\title{
Intelligente Instandhaltung
}

\author{
Präzision und Schnelligkeit sind wesentliche Qualitätsmerkmale für optische Beschichtungsprozesse. \\ Dabei sind moderne Vakuumkomponenten und -systeme, sowohl in der Fertigung als auch in der \\ Analytik, wichtige Voraussetzungen für wirtschaftliche und erfolgreiche Prozesse.
}

In optischen Beschichtungsprozessen bieten Schwingungsüberwachungssysteme für die installierten Pumpen und Systeme ein hohes Optimierungspotenzial. In dem breiten Spektrum moderner Vakuumlösungen kommen technologische Anwendungen von Leybold zum Einsatz. Da Vakuumsysteme ein zentraler Bestandteil des Herstellungsprozesses sind, erfordern sie zusätzlich intelligente Wartungsstrategien. Basierend auf einer Monitoring-Lösung bietet Leybold seinen Kunden daher bedarfsgerechte, maßgeschneiderte Service Level Agreements (SLA). Zudem garantiert das Unternehmen die Verfügbarkeit von Service-Mitarbeitern und schnelle Reaktionszeiten. Die zustandsorientierten, vorausschauenden Instandhaltungsstrategien von Leybold sichern Anwendern eine maximale Anlagenverfügbarkeit und minimieren gleichzeitig ungeplante, teure Produktionsausfälle.

Ein erhöhter Verschleiß an der Pumpe wird ebenso rechtzeitig erkannt wie die negative Beeinflussung von Prozessbedingungen, wie etwa Ablagerungen oder Partikeleintrag. Der positive Effekt: Wartungsmaßnahmen werden exakt dann, aber auch nur dann, eingeleitet, wenn der Zustand der Pumpe den Eingriff auch erfordert. Das Ergebnis: Die Laufzeiten werden dadurch maximiert und das Wartungspersonal kann zielgerichtet eingesetzt werden. Im Hinblick auf die Ausfallkosten bietet die vorbeugende Instandhaltung einen hohen Return on Investment (ROI) und sorgt zusätzlich für einen kontinuierlichen Verbesserungsprozess im gesamten Instandhaltungsmanagement und in der Produktion.
Vorausschauende Instandhaltungsstrategien sichern in optischen Beschichtungsprozessen eine maximale Anlagenverfügbarkeit und minimieren gleichzeitig ungeplante, teure Produktionsausfälle.

\section{Prognosen dauerhaft verbessern}

In den meisten Fällen benötigen Unternehmen nicht einmal die zusätzliche Installation eines teuren Sensors. In einem ersten Schritt werden lediglich die Daten gelesen und genutzt, die die Pumpen ohnehin produzieren. Zusätzlich können Sensoren zum Einsatz kommen, um die Prognosen bei komplexeren Aufgaben zu verbessern. Doch nicht nur die zustandsorientierte oder gar komplette Fernüberwachung optimiert den After Sales. Auch ein schneller und effektiver Service durch hochqualifizierte Servicetechniker und eine weltweite Verfügbarkeit von Original-Ersatzteilen oder -Kits sichern einen anwenderspezifischen Serviceansatz. Dieser wird ergänzt durch Features wie Backup-Pools oder Logistik-Konzepte.

Im Mittelpunkt steht vor allem die Systemverfügbarkeit. Die Qualität der Pumpen, gepaart mit zusätzlichen Serviceoptionen und dem Know-how der Servicemitarbeiter ermöglichen es, dem Anwender sehr schnell zu helfen und so Betriebskosten und Ausfallzeiten zu reduzieren. Ein optimiertes, geplantes Servicekonzept versetzt den Anwender in die Lage, sich auf

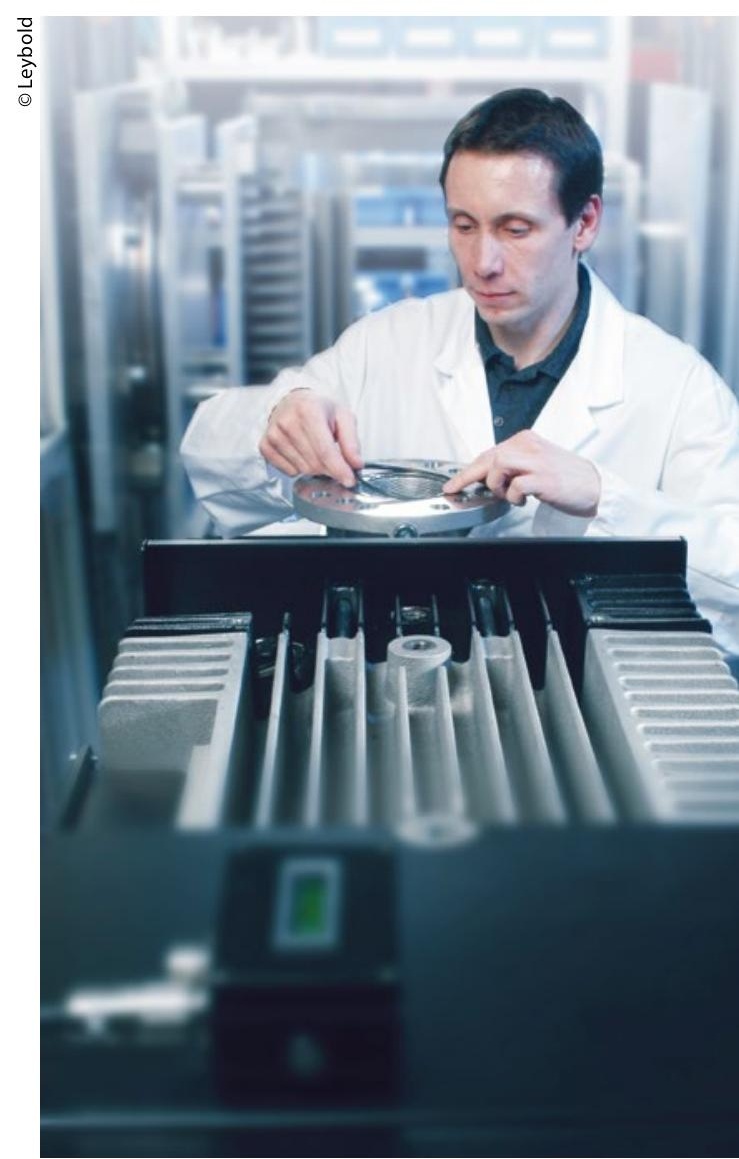

das Kerngeschäft zu konzentrieren. Dies ist eine der effektivsten Strategien, um den ROI zu erhöhen, insbesondere in der Serienfertigung. //

\section{Kontakt}

\section{Leybold GmbH}

Köln

Tel. 02213470

info@leybold.com,

www.leybold.com 